\title{
Rosacea fulminans with rhinophyma and severe eye complications in a young woman treated with isotretinoin
}

\author{
Joanna Pogorzelska-Dyrbuś ${ }^{1}$, Marek Sosnowski², Jacek C. Szepietowski ${ }^{3}$ \\ "'Estevita” Specialist Medical Practice, Tychy, Poland \\ 2Department of Ophthalmology, University Clinical Centre, Medical University of Silesia, Katowice, Poland \\ ${ }^{3}$ Department of Dermatology, Venereology and Allergology, Wroclaw Medical University, Wroclaw, Poland
}

Adv Dermatol Allergol 2021; XXXVIII (5): 903-905

DOI: https://doi.org/10.5114/ada.2020.94743

Rosacea is an inflammatory skin disease that affects the cheeks, nose, chin and forehead [1]. Rosacea fulminans is an extremely rare variant of rosacea, previously called pyoderma faciale, which usually occurs in healthy young women. It is characterised by an abrupt onset of erythematous papules, pustules, nodules and cysts on the background of - often severe - redness. The most worsened late stage of rosacea is called rhinophyma. The name, which was first used by Hebra in 1845, derives from the ancient Greek word "rhis", which means nose, and "phyma”, meaning growth [2].

Rhinophyma is a benign and slowly progressive nodular thickening of the nose, resulting from hypertrophy of the sebaceous glands and connective tissue. In contrast to rosacea fulminans, rhinophyma occurs almost exclusively in men, mostly between their 50s and 60s [3].

To the best of our knowledge, to date, there have only been a few published reports of this condition in women $[4,5]$. Here, we report a case of a female patient with rosacea fulminans leading to rhinophyma with coexisting dramatic ophthalmic complications.

A 31-year-old Caucasian woman presented with a 6-month history of rosacea fulminans and simultaneous rhinophyma. Clinical examination revealed a cauliflower-like deformity of the lower two-thirds of the nose. The patient had also manifested the central facial erythema and multiple papules, pustules, cysts and scars, but no comedones (Figure 1).

She gave a history of an abrupt, severe exacerbation of skin lesions for about 6 months without flushing. The patient did not report any inflammatory bowel diseases or endocrine disorders. Moreover, she had not suffered from acne in puberty. Before admission, the patient was treated only with antibiotic ointments. She also reported critical worsening of vision, close to blindness.

The patient was referred to an immediate ophthalmological examination, which revealed that both eyes were simultaneously affected by ocular complications of the disease; namely on the basis of exudative uveitis with the involvement of the retina and vitreous body, retinal ischaemia developed, resulting in neovascularization and subsequent vitreous haemorrhages. Hence, based on medical history and physical examination, a diagnosis of rosacea fulminans with rhinophyma and ocular involvement was made.

She was administered a daily course of isotretinoin $40 \mathrm{mg}(0.66 \mathrm{mg} / \mathrm{kg}) 4$ four months. According to the standard clinical practice, to accelerate the treatment, the dose of the drug after that time was increased to daily $50 \mathrm{mg}$ for 3 months, and finally, having achieved a satisfying result, reduced to daily $30 \mathrm{mg}$ for 6 months to maintain a proper outcome. In the meantime, the patient underwent bilateral posterior vitrectomy with silicone oil endotamponade followed by daily $4 \mathrm{mg}$ methylprednisolone treatment for 3 weeks.

After thirteen months of isotretinoin treatment, an excellent overall improvement was noted, papules, pustules and cysts were completely cured and the normal contour of the nose was restored (Figure 2). The patient reported a significant restitution of vision. No recurrence of any symptoms of rosacea was noticed within 8 months of follow-up. The patient's well-being improved considerably.

The etiopathogenesis of rosacea is still not clear. It is believed that oxidative stress, decreased superoxide dismutase production of vasoactive mediators, such as

Address for correspondence: Prof. Jacek C. Szepietowski MD, PhD, Department of Dermatology, Venereology and Allergology, Wroclaw Medical University, 1 Chalubinskiego St, 50-368 Wroclaw, Poland, e-mail: jacek.szepietowski@umed.wroc.pl Received: 7.01.2020, accepted: 3.02.2020. 


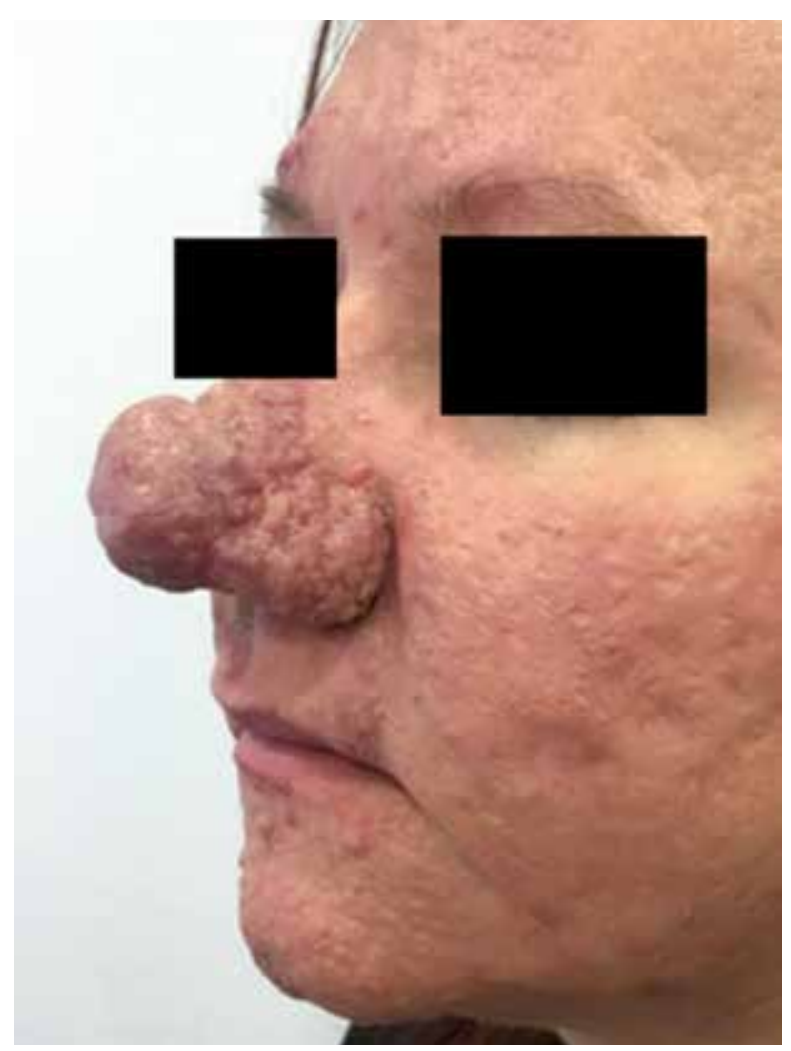

Figure 1. Baseline clinical presentation of the patient

substance $P$, serotonin and prostaglandins, may play an important role in its development [6].

Although women are about 3 times more likely to be affected by the facial manifestations of rosacea, men more often display rhinophyma and their disease is more severe [5]. Higher prevalence of nose deformity among men may be partially explained by the influence of androgens [7]. A literature review revealed only a few cases of rhinophyma in women, generally much older than our patient, who was in her early 30s [4, 5].

The clinical features of rosacea fulminans may mimic acne fulminans, but ocular complications could be a helpful suggestion in the differential diagnosis of both disorders as ophthalmic complications do not occur in cases of acne fulminans. Moreover, acne is rarely confined to the face and is characterised by the presence of comedones. According to the literature, rosacea fulminans usually occurs in young healthy women, which is consistent with the age of our patient $[4,8]$.

Ocular manifestations of rosacea are seen in 3-58\% of cases with a slightly higher occurrence in men than in women [9]. The common ophthalmological changes include blepharitis, conjunctivitis, meibomian gland dysfunction and chalazia. Patients usually complain of itching, dryness and foreign body sensation. Scleritis, episcleritis, corneal thinning and neovascularization and blindness occur very rarely. Although ocular rosacea is

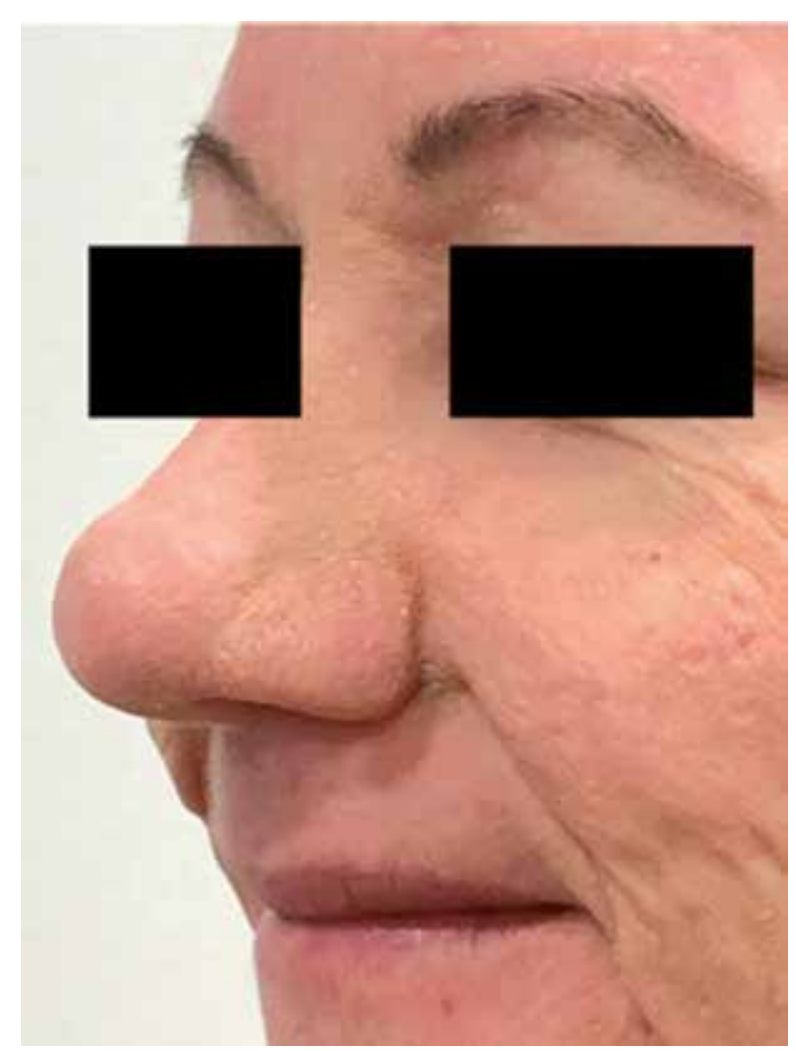

Figure 2. Clinical presentation of the patient after treatment

not an uncommon finding, no cases with such severe eye complications, including dramatic vision impairment developing in the mechanism of choroideal inflammation and neovascularization with vitreous haemorrhages, like in our patient, have been reported so far.

Our case represents the multidisciplinary approach to the treatment of rosacea, with a parallel involvement of the dermatologist and the ophthalmologist. Although retinoic acid derivatives, including isotretinoin, are known to impair healing, the surgical ophthalmological treatment was performed without reduction in the dose of the drug. The rationale for such decision was that ocular conditions of the patient were solely the result of the underlying rosacea.

For many years, both medical and surgical treatments have been used to manage rhinophyma with varying results. Nowadays surgical excision is still the mainstay of treatment, but isotretinoin is also capable of reducing rhinophyma [10]. With regards to rosacea fulminans, the majority of the data support the use of systemic corticosteroids and isotretinoin as the most effective ways of treatment [11]. To our knowledge, only a few cases of successful pharmacological treatment of such a severe stage of rosacea have been reported and therapy was often long-lasting with its results being generally disappointing [7]. It should be noted that our patient's response to pharmacological treatment modalities without a need 
for surgical intervention was surprisingly sufficient. No recurrence of any symptoms of rosacea was noticed in the 8-month follow-up.

In conclusion, although rosacea with ocular complications and rosacea with rhinophyma have been reported, the coexistence of rhinophyma and such severe ocular complications in a woman has not been reported so far.

\section{Conflict of interest}

The authors declare no conflict of interest.

\section{References}

1. van Zuuren EJ, Rosacea. N Engl J Med 2017; 377: 1754-64.

2. von Hebra F. Versuch einer auf pathologische Anatomie gegründeten Einteilung der Hautkrankheiten Z. Gesellschaft Ärzte Wien 1845; 2: 211.

3. Rørdam OM, Guldbakke K. Rhinophyma: big problem, simple solution Acta Derm Venereol 2011; 91: 188-9.

4. Mahajan VK, Sharma AL, Chauhan PS, et al. SkIndia Quiz 27: Rhinophyma and numerous facial papule and nodules in a 39-year-old woman. Indian Dermatol Online J 2016; 7 : 350-2.

5. Odou BL, Odou ER. Rhinophyma. Am J Surg 1961; 102: 3-16.

6. Crawford GH, Pelle MT, James WD. Rosacea: I. Etiology, pathogenesis, and subtype classification. J Am Acad Dermatol 2004; 51: 327-41.

7. Sadick H, Goepel B, Bersch C, et al. Rhinophyma: diagnosis and treatment options for a disfiguring tumor of the nose. Ann Plast Surg 2008; 61: 114-20.

8. Kim TG, Noh SM, Do JE, et al. Rosacea fulminans with ocular involvement. Br J Dermatol 2010; 163: 877-9.

9. Akpek EK, Merchant A, Pinar V, Foster CS. Ocular rosacea: patient characteristics and follow-up. Ophthalmology 1997; 104: 1863-7.

10. Little SC, Stucker FJ, Compton A, Park SS. Nuances in the management of rhinophyma. Facial Plast Surg 2012; 28: 231-7.

11. Jansen T, Plewig G, Kligman AM. Diagnosis and treatment of rosacea fulminans. Dermatology 1994; 188: 251-4. 